\title{
La triple narrativité du MMORPG à travers la pratique du roleplay
}

\section{Géraldine Wuyckens}

\section{(2) OpenEdition \\ Journals}

Édition électronique

URL : http://journals.openedition.org/sdj/1032

DOI : $10.4000 /$ sdj. 1032

ISSN : 2269-2657

Éditeur

Laboratoire EXPERICE - Centre de Recherche Interuniversitaire Expérience Ressources Culturelles Education

\section{Référence électronique}

Géraldine Wuyckens, "La triple narrativité du MMORPG à travers la pratique du roleplay », Sciences du jeu [En ligne], 9 | 2018, mis en ligne le 21 juin 2018, consulté le 20 avril 2019. URL : http:// journals.openedition.org/sdj/1032 ; DOI : 10.4000/sdj.1032

Ce document a été généré automatiquement le 20 avril 2019

Tous droits réservés 


\title{
La triple narrativité du MMORPG à travers la pratique du roleplay
}

\author{
Géraldine Wuyckens
}

1 La narratologie est une discipline se consacrant à l'étude du récit. Elle trouve son origine dans les études littéraires, ce qui explique son intérêt premier pour les récits sous la forme de textes écrits et organisés par un auteur. Depuis une vingtaine d'années, ses objets d'études s'élargissent, intégrant notamment la problématique de l'interactivité. Des auteurs comme Umberto Eco (1984), Marie-Laure Ryan (2001) ou Hilary Danneberg (2004) prennent ainsi en compte le rôle du lecteur dans la configuration du récit et la construction de mondes fictionnels (Marti et Baroni, 2014). L'apparition de fictions interactives oblige toutefois à redéfinir certains concepts de narratologie et à confronter celle-ci à d'autres disciplines pour comprendre les nouvelles configurations que peuvent prendre les récits actuels.

2 Concernant le jeu vidéo, la narratologie littéraire est «intéressante pour aborder l'analyse de l'intrigue dans le jeu vidéo car elle prend en compte non seulement la création et le fonctionnement de la structure matérielle du jeu mais aussi l'expérience narrative qu'elle suppose » (Marti, 2014, p. 3). L'étude de la structure matérielle du jeu s'intéresse au jeu tel que défini par un système de règles. Elle se penche sur l'histoire préprogrammée et préétablie du jeu vidéo, soit la narrativité «statique». Le jeu vidéo repose sur une seconde narrativité, produite par l'interaction du joueur avec le jeu. Celleci prend forme via « les modalités d'action laissées à disposition du joueur pour agir dans l'œuvre» (Genvo, 2009, p. 142), qui font de celui-ci à la fois le récepteur et l'auteur du récit. Cette « double » narrativité constitue la narrativité vidéoludique, autrement dit « la combinaison entre une intrigue "préprogrammée" et matérielle, et l'expérience de cette intrigue par le joueur» (Marti, 2014, p. 2). Elle renvoie aux deux formes principales du récit du jeu vidéo étudiées par plusieurs chercheurs en narratologie, comme le récit figé et le récit émergent de Selim Krichane (2014).

3 La narrativité vidéoludique peut être observée dans les MMORPGs, les jeux vidéo de rôle en ligne massivement multijoueur. Non seulement ceux-ci se caractérisent par une histoire très riche, communément appelée le Lore, mais ils offrent également aux joueurs 
de multiples possibilités d'interaction avec le jeu. Reprenant les résultats d'une recherche menée dans le cadre d'un mémoire de fin d'études en 2016 (Wuyckens, 2016), le présent article se consacre à l'étude des différentes narrativités du MMORPG et à la configuration spécifique que celles-ci peuvent prendre en relation avec un esprit communautaire. Il se penche pour cela particulièrement sur la pratique du roleplay ${ }^{1}$ (en français, le jeu de rôle) qui consiste, dans un jeu vidéo en ligne, à s'exprimer comme le ferait son personnage, et non en tant que joueur, avec les autres personnages de la communauté des joueurs. Cette pratique se caractérise par une immersion narrative du joueur par le récit de la communauté des rôlistes, voire celui de la guilde à laquelle il appartient. Sur la base des résultats d'une ethnographie virtuelle réalisée dans le MMORPG World of Warcraft (WoW), l'article tente de comprendre comment les rôlistes négocient avec les différentes couches narratives du jeu via l'interprétation de leur(s) personnage(s) et leur interaction avec les autres personnages des joueurs de la communauté.

\section{Trois types de narrativité au sein du MMORPG}

4 Les paragraphes suivants se consacrent à l'étude de l'architecture narrative du MMORPG. Trois narrativités sont proposées : la narrativité préétablie par les concepteurs du jeu, la narrativité issue de l'interaction du joueur avec le jeu et la narrativité créée par la communauté des joueurs comprenant notamment les histoires issues de la pratique du roleplay. Le concept de " narrativité ", tel qu'utilisé dans cet article, renvoie à tout ce qui relève du récit : les histoires, et ce, peu importe leur forme et leur support (textes écrits, dessins, captures d'écran, forums, films, etc.). Cette notion est privilégiée à celle de «narration ", entendue comme l'acte de raconter une histoire : la narrativité fait quant à elle référence aux caractéristiques formelles du récit.

5 Tout d'abord, au cours de l'exploration dans le jeu, les joueurs suivent « un fil rouge narratif qui traverse et anime ces univers, ce qu'ils nomment le "background" ou la " storyline", autrement dit l'histoire globale qui concerne tout l'univers de référence " (Berry, 2012a, p. 97). Lors de la conception de l'avatar, le joueur choisit en général un clan, une race et une profession pour son personnage, autant de catégories reposant chacune sur une histoire. Ces choix axent l'histoire même du personnage dans le jeu et l'inscrivent dans une histoire plus globale. Par exemple, dans Guild Wars 2 (ArenaNet, 2012), le joueur doit sauver la Tyrie des dragons ancestraux. Afin d'y parvenir, il doit réaliser un ensemble de quêtes données par des personnages non-joueurs (ou PNJs). Sous forme de petites histoires, les quêtes participent au récit global du jeu. Dans son analyse de produits multimédia, Frédéric Dajez nomme «ludo-narration [... cet] enchainement d'épreuves ludiques dans un récit global qui donne sens »(Dajez, 2006, p. 23).

Bien souvent, l'histoire globale ne s'arrête pas aux seules frontières du jeu. Les concepteurs développent celle-ci sur de nombreux autres supports, comme le site internet du jeu, des figurines, des jeux de rôle sur plateau, des films ou encore des jeux de cartes. Les MMORPGs sont par conséquent des exemples de narration transmédiatique, celle-ci se déployant sur de multiples plateformes médiatiques, chaque texte étant nouveau et apportant sa contribution à l'histoire globale (Jenkins, 2013). En plus d'être multi-supports, l'univers narratif est multi-récits: le jeu propose une multiplicité de récits réunis dans un même univers, et non pas un seul récit canonique. Par ces caractéristiques, le MMORPG peut être qualifié d'" univers narratif transmédiatique ", c'est-à-dire un ensemble organisé d'histoires se développant sur plusieurs médias et 
formant un tout. Dans un article se consacrant à l'architecture narrative du jeu, Henry Jenkins (2002) utilise le concept de « narration environnementale » (traduit de l'anglais « environmental storytelling ") pour désigner les histoires des concepteurs du jeu. Le récit figé du MMORPG inclurait par conséquent l'histoire véhiculée par le jeu (les caractéristiques des régions et des personnages, les quêtes données par les PNJs ou encore les didacticiels), mais également les informations narratives provenant d'autres plateformes médiatiques et contribuant au Lore.

7 Ensuite, la narrativité issue de l'interaction du joueur avec le jeu comprend les contenus narratifs actualisés par le joueur via son expérience dans le jeu: elle fait référence au récit vidéoludique, «qui est le résultat d'une suite d'actions, réalisées par le joueur lors d'une partie, de ses choix de parcours» (Barnabé, 2012, p.15). Elle se manifeste principalement par l'interaction du joueur avec les quêtes du jeu: en réalisant ces missions, le personnage acquiert de l'expérience, des compétences, des équipements et devient de plus en plus puissant. Il progresse jusqu'à devenir un héros dont la mission est de sauver le monde. Ce mode de jeu est reconnu par la communauté des joueurs comme le "mode divin", signifiant que le joueur incarne une sorte de Dieu. Ce mode renvoie à l'aspect RPG (roleplaying game) du MMORPG où chaque joueur est amené à jouer un rôle à travers son personnage. Dans cette première forme d'interaction, la première et la seconde narrativité se croisent et le rôle du récit figé va être d'inscrire le joueur dans une histoire globale et logique afin qu'il puisse faire progresser son personnage dans le jeu.

8 Les MMORPGs offrent de nombreuses autres possibilités aux joueurs. Dans sa théorie des prototypes narratifs, Marc Marti (2014) souligne que, contrairement aux autres jeux vidéo qui proposent un dénouement à la fin de leur histoire, les jeux évolutifs, dont le MMORPG,

s'éloignent des modèles canoniques du récit, en particulier si l'on examine la position qu'y occupe le joueur. Celui-ci n'est plus « guidé » par une trame narrative (unique ou multiple) d'enchaînement des niveaux ou des épreuves jusqu'à un climax final qui constituerait la «fin» théorique du jeu et est au contraire en grande partie laissé libre grâce aux possibilités nombreuses d'interactions qui lui sont offertes (Marti, 2014, p. 8).

9 En effet, dans le MMORPG, le joueur est non seulement invité à avancer dans l'histoire du jeu à travers l'accomplissement de diverses quêtes, mais également à entrer dans une guilde, à combattre d'autres joueurs dans des arènes de JcJ (Joueur contre Joueur), à obtenir tous les titres et succès, ou encore à participer aux événements spéciaux proposés par le jeu. L'interaction du joueur avec le jeu ne se résume donc pas à l'accomplissement des quêtes préprogrammées et varie énormément d'un jeu à l'autre. Chaque joueur est ainsi amené à construire sa propre histoire.

10 Malgré la grande liberté que permet la seconde narrativité, le récit vidéoludique reste fort dépendant de la structure du jeu car il ne prend forme qu'à partir des actions rendues possibles par le jeu. Or, certains joueurs ne s'arrêtent pas aux interactions avec le jeu et développent des activités qui n'étaient pas initialement prévues par les concepteurs. Ces dernières s'inscrivent dans le méta-jeu, regroupant les activités se constituant autour du jeu (Berry et Brougère, 2001, p. 8) : les fanfictions, les communautés de joueurs (les guildes), les sites internet et forums, les activités de capitalisation (vente, achat, collection) ou encore les rencontres des joueurs dans la vraie vie. Tout comme celles proposées par le jeu, ces activités sont génératrices d'histoires et sont issues des créations des joueurs et de leurs interactions avec les autres joueurs. Elles constitueraient 
par conséquent une «troisième narrativité » qui caractériserait le MMORPG d'une triple narrativité.

11 Loin de former un récit unique, la troisième narrativité est composée d'un ensemble de récits contribuant les uns aux autres. Ceux-ci peuvent être construits individuellement ou collectivement, en jeu ou en dehors du jeu. Ce qui fait la spécificité de la narrativité du MMORPG, et le distingue des jeux vidéo hors ligne, concerne surtout les récits construits collectivement, cela étant rendu possible par son aspect MMO. Par sa création constante de récits par les joueurs, le roleplay est une activité s'inscrivant dans la troisième narrativité. Bien que cette pratique repose essentiellement sur le jeu, via l'interprétation de son personnage, elle n'est pas initialement prévue par les éditeurs de jeu et serait donc de l'ordre du méta-jeu. Il ne s'agit toutefois pas d'une activité « méta » au sens propre du terme, le roleplay ne visant pas à surplomber l'univers du jeu mais plutôt à prendre appui sur celui-ci pour développer ses propres histoires. Le roleplay invite par conséquent à repenser le modèle théorique du méta-jeu. Dans la perspective narrative telle qu'adoptée dans cet article, le méta-jeu comprend les « autours du jeu », défini par Berry et Brougère (2001), ainsi que des activités qui ne se situent pas forcément au-dessus jeu, comme le roleplay ou les machinimas (petits films tournés en jeu).

\section{La pratique du roleplay}

12 Tirant ses origines du jeu de rôle sur table, le roleplay est une pratique qui consiste à incarner un personnage comme un acteur le ferait dans une pièce de théâtre ou au cinéma. Plus précisément :

Faire du roleplay revient à s'inventer un personnage cohérent vis-à-vis du monde de jeu et à le faire vivre. Le fait de jouer ce personnage peut être conçu comme une imitation. [...] La pratique du roleplay se trouve à mi-chemin entre écriture et jeu d'acteur et varie énormément en terme d'intensité et de style en fonction des joueurs, des communautés et des jeux (Lelièvre, 2012b, p. 98).

13 En se rapportant à la ludologie, le roleplay peut être rattaché au mimicry, défini par Roger Caillois comme l'ensemble varié des «manifestations qui ont pour caractère commun de reposer sur le fait que le sujet joue à croire, à se faire croire ou à faire croire aux autres qu'il est un autre que lui-même » (Caillois, 1967, p. 61). Le jeu repose sur cette illusion, où le sujet joue à être autre, à devenir lui-même un personnage illusoire et à se conduire comme celui-ci. « Tout jeu suppose l'acceptation temporaire, sinon d'une illusion (encore que ce dernier mot ne signifie pas autre chose qu'entrée en jeu : in-lusio), du moins d'un univers clos, conventionnel et, à certains égards, fictif » (p. 60).

Tels des comédiens, les rôlistes en ligne jouent un rôle, celui du personnage qu'ils incarnent. Ils suspendent volontairement leur incrédulité et acceptent l'illusion pour entrer dans la fiction (Coleridge, 1817). Afin qu'il y ait roleplay, ils font semblant de croire que c'est vrai, même s'ils savent bien qu'il s'agit d'un jeu second. Deux types d'activités sont principalement réalisées par les rôlistes des MMORPGs, en jeu et hors-jeu ; celles-ci ne sont pas prévues dans le système initial du jeu, si bien qu'elles se rattachent plutôt au paidia (divertissement, improvisation, fantaisie) qu'au ludus (règles, paramètres formels du jeu) (Caillois, 1967). D'une part, le roleplay consiste à organiser des événements avec des scénarios conçus par les joueurs et à créer des règles supplémentaires au-dessus de celles du jeu. D'autre part, les rôlistes inventent des histoires en dehors du jeu, comme 
des nouvelles, des fanfictions ou des historiques (backgrounds) liant ou non les personnages entre eux (Lelièvre, 2012a, pp. 4-5).

\section{Méthodologie : une ethnographie virtuelle}

Afin d'étudier la pratique du roleplay, la méthode de l'ethnographie virtuelle a été mobilisée. «À un niveau très général, cette notion se définit comme l'étude des populations et des pratiques sur le réseau mondial : tchats, forums de discussions, sites internet, blogs, réseaux sociaux ... et mondes virtuels » (Berry, 2012b, p. 36). Apparue dans la seconde moitié des années 90, l'ethnographie virtuelle est une approche qualitative, consistant pour l'ethnologue à s'immerger dans un terrain virtuel afin d'étudier les interactions entre les différents acteurs en son sein. "À la manière d'un ethnographe qui autrefois arpentait de lointaines contrées, muni de son carnet de notes, le chercheur devrait donc, à notre sens, explorer ces univers avec la même rigueur " (Trémel, 2003, p. 167).

16 Afin de de mener à bien cette étude, j'ai pris exemple sur les travaux des chercheurs anglo-saxons Tom Boellstorff (Second Life), Bonnie Nardi (World of Warcraft), Celia Pearce ( Uru: Ages Beyond Myst) et T.L. Taylor (Everquest) qui ont tous les quatre investis des mondes virtuels (Boellstorff et alii, 2012 ; Boellstorff, 2013 ; Nardi, 2010; Pearce, 2011; Taylor, 2009). Dans leur ouvrage collectif et leurs ouvrages respectifs, ils fournissent des techniques pratiques et détaillées pour la recherche ethnographique dans les mondes virtuels qui m'ont guidées tout au long de mon travail : leurs conseils méthodiques pour la préparation au terrain et la collection des données m'ont particulièrement aidés. Du côté francophone, la lecture de Vincent Berry (2012a, b), Laurent Trémel (2003), Olivier Servais $(2012$; 2013) et Etienne Armand Amato (2007) m’a également guidée pour les différentes étapes de mon immersion participante et la réalisation des questionnaires en ligne.

\section{L'immersion participante}

17 La méthode principale de l'ethnographie classique est l'observation participante, consistant pour le chercheur à devenir acteur (et non seulement spectateur) des activités quotidiennes se déroulant dans le terrain étudié. Dans le cadre d'une ethnographie virtuelle, les chercheurs ont généralement recours à l' " immersion participante », où il s'agit d'expérimenter par soi-même « les conditions de participation vécues par tous les participants à l'intérieur du jeu» (Amato, 2007). Afin de comprendre les pratiques des joueurs, il me semblait important de ne pas seulement les observer mais d'y participer, et par conséquent d'adopter ladite méthode de l'immersion participante. Comme Bonnie Nardi (2010) l'a fait pour étudier les raids dans WoW en apprenant elle-même à devenir un «raider", j'ai donc décidé de devenir rôliste pour étudier la pratique. "Être directement impliqué dans les activités quotidiennes fournit une vision intime de leur substance et de leur sens" (Boellstorff et al., 2012, p.65, ma traduction). Lors de l'immersion participante, d'autres données peuvent être récoltées; dans le cadre de ce travail, j'ai enregistré des extraits du chat conversationnel et pris des captures d'écran afin d'illustrer et d'expliquer au mieux la pratique communautaire du roleplay dans le jeu concerné. 
18 Après avoir parcouru de nombreux mondes virtuels pendant plusieurs mois à la recherche de communautés de rôlistes, mon choix s'est arrêté au jeu World of Warcraft ( WoW). Il a été sélectionné pour trois principales raisons. Premièrement, la richesse de son Lore qui comprend «les écrits et conceptions de Blizzard au sujet du jeu, et ce, sur plusieurs supports : jeu vidéo (WoW, Warcraft), livres, comic books, vidéos. Elle [l'Histoire de WoW] retrace tous les événements ayant pris place dans l'univers de Warcraft et d'Azeroth»(Millénium, 2015). Deuxièmement, la communauté des joueurs de WoW développe une culture spécifique à travers la formation de collectivités virtuelles, les guildes. Ces microgroupes sociaux forment une « culture tribale virtuelle » (Servais, 2012, p. 235). Les guildes développent en effet leurs propres normes, coutumes, croyances, histoires, pratiques et s'attribuent des objectifs dans le jeu. Elles constituent une composante essentielle du jeu assurant la réussite des joueurs. Dans les serveurs de JdR (Jeu de Rôle), des guildes sont dédiées exclusivement au roleplay, ce qui est d'autant plus intéressant dans le cadre de cette recherche. Troisièmement, le roleplay est une pratique encore assez courante dans le jeu. Dans les serveurs de JdR, il est possible de croiser des joueurs faisant du roleplay dans des lieux publics, tels que des places, des auberges, ou à l'occasion d'événements.

Concernant le choix du serveur, Kirin Tor a été sélectionné car il s'agit du tout premier serveur JdR francophone créé par Blizzard en 2005 et il est composé d'une vieille communauté de rôlistes, certains d'entre eux faisant le jeu de rôle depuis plus de sept ans. L'immersion participante s'est déroulée durant trois mois, de février à avril 2016, pour un total de 70h de jeu. Au cours de celle-ci, j'ai intégré la communauté des rôlistes de Kirin Tor : j'ai créé divers avatars, appris à pratiquer le roleplay et intégré deux guildes. Pour me faire pleinement acceptée par la communauté, je suis également passée par la création d'un historique de mon personnage, l'apprentissage des règles et la participation aux événements RP. J'ai évité au maximum l'observation cachée. Comme Boellstorff et al. (2012) l'attestent,

20 il est important de souligner qu'en tant qu'ethnologues, nous sommes obligés de révéler à nos informateurs autant que possible la nature et le but de nos études. Fournir des informations concernant les objectifs et les méthodes est nécessaire pour établir et maintenir de bonnes relations (Boellstorff et al., 2012, p. 133, ma traduction).

21 Par sa définition, le roleplay implique néanmoins un certain camouflage de la personne derrière l'écran. Lorsque j'ai réalisé du roleplay dans le jeu, je n'ai ainsi pas toujours informé mes interlocuteurs que j'étais chercheuse, cela faisant partie du domaine du HRP (hors-roleplay ${ }^{2}$ ). De plus, informer les joueurs comportait un risque de biaiser l'immersion participante puisqu'ils n'auraient peut-être pas interprété leur personnage de la même manière si je ne leur avais rien révélé. J'ai donc communiqué mes intentions pour les relations à plus longue durée. Révéler son statut comporte certains avantages : «Non seulement les joueurs ne sont jamais (ou rarement) hostiles aux études sur les MMO (sauf si elles concernent la question de l'addiction et à la condition d'être soi-même joueur), ils sont souvent curieux, parfois enthousiastes " (Berry, 2012b, p. 46). Je n'ai rencontré de ce fait aucune difficulté à convaincre des joueurs de répondre à mes questions.

\section{Le questionnaire}

22 La méthode de l'immersion participante est généralement accompagnée d'interviews individuelles et/ou en groupe, en jeu ou dans le monde réel, afin d'obtenir plus de 
données sur les joueurs. "La recherche sur les mondes virtuels peut recourir efficacement aux méthodes d'élicitation, mais se doit également de les dépasser pour élaborer des méthodes basées sur la technè, et pas uniquement sur l'épistémè » (Servais, 2013, p. 134). Dans le cadre de cette recherche, l'immersion participante est accompagnée de questionnaires afin de tenter d'aboutir à une généralisation de la pratique communautaire du roleplay dans le serveur concerné et d'établir un lien entre ce que les joueurs disent et font.

l'utilisation du questionnaire en ligne est apparue comme une nécessité, pour saisir la diversité de la population, que l'ethnographie et les processus d'homophilie permettent difficilement de saisir: âge, catégorie socioprofessionnelle, capital culturel, économique, temps de jeu, fréquence, pratiques culturelles parallèles, etc. (Berry, 2012b, p. 51).

La combinaison de ces deux méthodes de recueil de données renvoie à la triangulation des données, définie par Marie-Fabienne Fortin (1966) «comme l'emploi d'une combinaison de méthodes et de perspectives permettant de tirer des conclusions valables à propos d'un même phénomène » (p. 318). La triangulation a pour objectif d'apporter une meilleure compréhension du phénomène étudié et de réduire les biais.

À destination des rôlistes du serveur de Kirin Tor, le questionnaire a été conçu une fois l'immersion participante terminée, celle-ci ayant permis d'avoir une meilleure idée des questions à poser aux rôlistes et par conséquent de mieux cadrer les sujets à aborder dans le questionnaire. À travers 43 questions comprenant des échelles linéaires, des questions ouvertes et des questions à choix multiples, les joueurs ont été interrogés sur leurs pratiques de WoW (leurs habitudes de jeu, leurs préférences), leur(s) personnage(s), leur pratique du roleplay et leur éventuelle appartenance à une guilde RP (guilde réalisant du roleplay). Après un prétest réalisé sur une dizaine de joueurs, il a été partagé sur le forum de battle.net (plateforme internet de Blizzard) et de la communauté RP du serveur. Méfiants au départ, il a fallu instaurer un climat de confiance avec les joueurs qui avaient peur que leurs réponses ne soient utilisées en leur défaveur pour dénigrer la pratique du roleplay. Mis en ligne durant 10 jours, un total de 81 réponses a été collecté, un nombre satisfaisant étant donné la faible fréquentation du jeu durant la période d'observation (beaucoup de joueurs avaient déserté le jeu, en attente de la sortie de la nouvelle extension Legions), qu'il s'agit d' un serveur francophone et que le questionnaire constitue une méthode complémentaire à l'immersion participante.

À titre informatif, 63 des enquêtés sont des hommes et 18 des femmes. La moyenne d'âge est de 26 ans. Quant au statut professionnel, presque la moitié des répondants sont des étudiants et 27 sont des employés. Les loisirs dominants sont les jeux vidéo pour 74 d'entre eux, les jeux de rôle pour 66 , le cinéma pour 55 , la lecture de romans pour 45 et les créations artistiques pour 34. En ce qui concerne leur pratique de jeux vidéo, ils jouent principalement à des jeux multijoueur en ligne ( 78 d'entre eux), des jeux de rôle/ d'aventures (66) et jeux de stratégie (55). Enfin, la majorité évalue l'intégration des jeux vidéo dans leur vie quotidienne comme assez forte, voire intensive pour un tiers d'entre eux. La population est donc relativement jeune, la majorité est des hommes, étudiants ou employés, ayant l'habitude de jouer à des jeux vidéo et à des jeux de rôle. Ce profil correspond aux joueurs rencontrés lors de l'immersion participante. 


\section{Le roleplay dans WoW : une pratique écrite et autonome}

fiay organisé. Ils se distinguent du fait que le premier est totalement improvisé, non prévu, contrairement au second qui a généralement lieu lors d'événements de la communauté ou d'une guilde. Concrètement, la pratique du roleplay se manifeste principalement par le texte et les actions des personnages. Le texte inclut les échanges écrits entre les joueurs interprétant leurs personnages. Quant aux actions, elles renvoient aux commandes prédéfinies par le système du jeu et à l'emploi d'astérisques, qui décrivent des actions ou des comportements exécutés par le personnage du joueur (par exemple, dans l'extrait de la conversation ci-dessous, «D-KirinTor lève les yeux vers elle » est une commande d'action). Certaines commandes sont associées à une animation ("/dance» écrit dans la fenêtre du chat fait danser le personnage) ou à une voix; d'autres non, si bien qu'il faut les imaginer et faire comme si le personnage les avait vraiment réalisées (c'est le cas des commandes en souligné repris dans l'exemple cidessous). Les commandes prennent la forme d'un texte écrit à la troisième personne du singulier, à l'image des didascalies au théâtre. Elles enrichissent de ce fait considérablement les discussions entre les joueurs en leur permettant d'agir en plus de parler.

Tableau 1. Extrait anonyme de conversations entre rôlistes

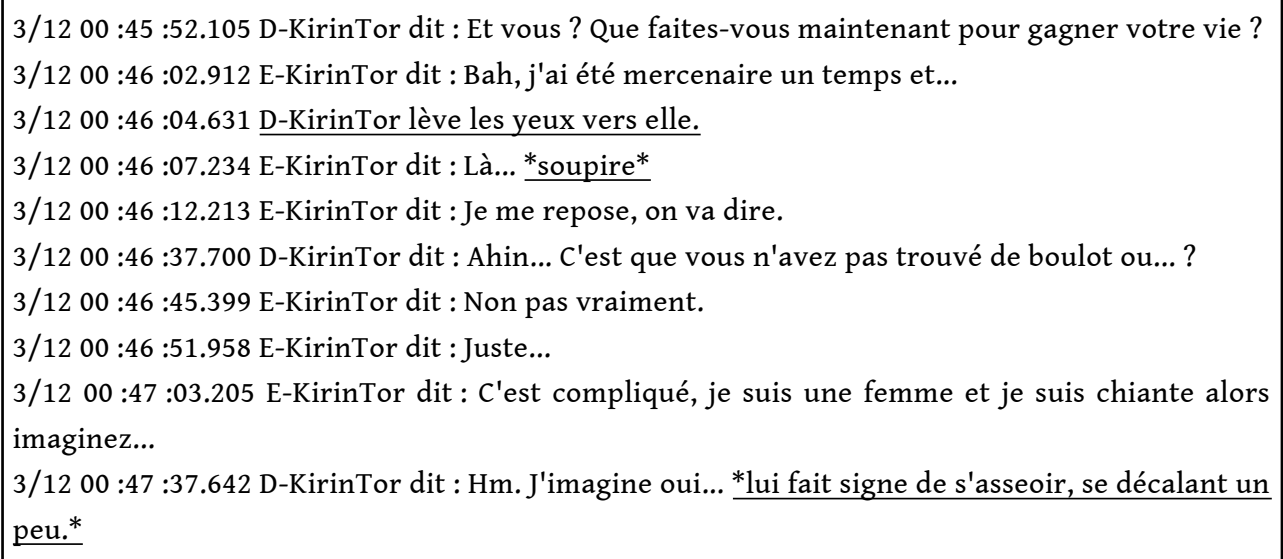

Comme l'illustrent les commandes d'action et l'emploi d'astérisques, l'imagination constitue une composante essentielle de la pratique. Les rôlistes essayent toutefois autant que possible de rendre leur jeu plus crédible à travers l'apparence et la position de leur personnage dans le jeu. À partir des observations menées en jeu, il semblerait, d'une part, 
que la plupart des joueurs utilisent des avatars de haut niveau pour le roleplay mais, peu importe leur expérience, ils peuvent être vêtus de manière très sobre, sans armures légendaires ou armes impressionnantes. Cela implique que si le joueur interprète un vagabond, son personnage devra non seulement s'exprimer comme tel mais également y ressembler physiquement. D'autre part, les joueurs semblent se parler toujours l'un en face de l'autre et évitent de se placer au même endroit qu'un autre joueur ou de traverser les avatars des autres quand ils se déplacent.

Les rôlistes se limitent de cette façon encore plus dans leur jouabilité. Par exemple, un joueur ne peut pas traverser un mur à cause de la structure du jeu. En roleplay, cela peut aller plus loin: le joueur fera la file derrière d'autres joueurs pour entrer dans un bâtiment alors que rien ne l'empêche de passer à travers tout le monde et d'y pénétrer (cf. Figure 1). Les actions limitées par la structure du jeu se distinguent par conséquent des actions limitées par le roleplay, dans un souci de vraisemblance. Nous voyons dès lors l'activité des rôlistes qui consiste à créer des règles supplémentaires qui se placent audessus de celles du jeu.

Figure 1

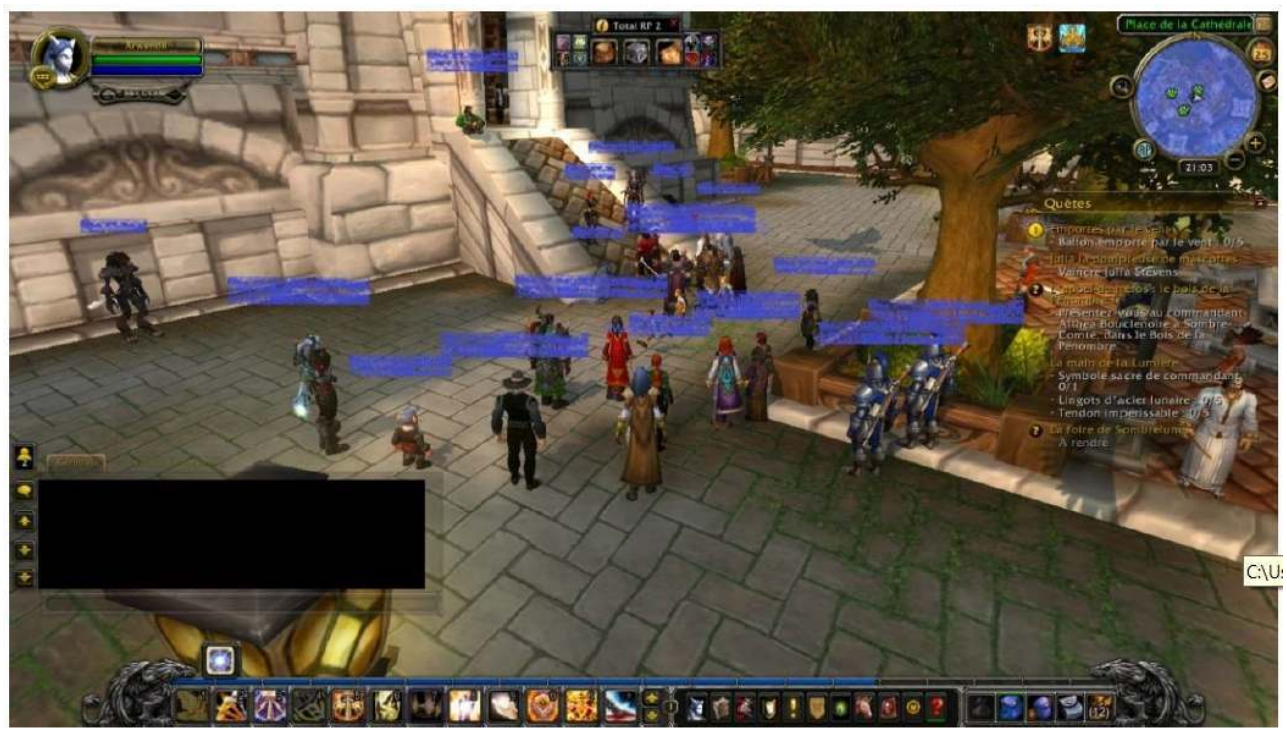

La file devant l'orphelinat d'Hurlevent (capture d'écran de l'auteur)

\section{La construction et l'interprétation de son personnage :}

\section{jouer avec les trois narrativités}

La création d'un personnage passe généralement par la réalisation d'un historique. Afin de constituer l'histoire de celui-ci, la majorité des rôlistes de Kirin Tor semblent s'inspirer du Lore de WoW. Ils découvrent le Lore à travers leur progression dans le jeu ainsi qu'en lisant les romans de Warcraft, en parcourant les forums ou en discutant avec les autres rôlistes dans le jeu. Environ 80 \% des répondants du questionnaire admettent ainsi que l'histoire de leur personnage est liée à celle du jeu, et même totalement mêlée à celle-ci pour la moitié d'entre eux. D'autres sources d'inspiration sont fortement utilisées en 
complément du Lore du jeu : la Fantasy pour 55 d'entre eux, l'Histoire pour 44 ainsi que la religion et la mythologie (celtique, germanique) pour 39.

Dans WoW, les joueurs ont la possibilité de créer des modules complémentaires au jeu. Le module «Total RP» (cf. Figure 2), créé par un rôliste du serveur, propose de créer diverses fiches décrivant le personnage qu'on interprète.

Figure 2

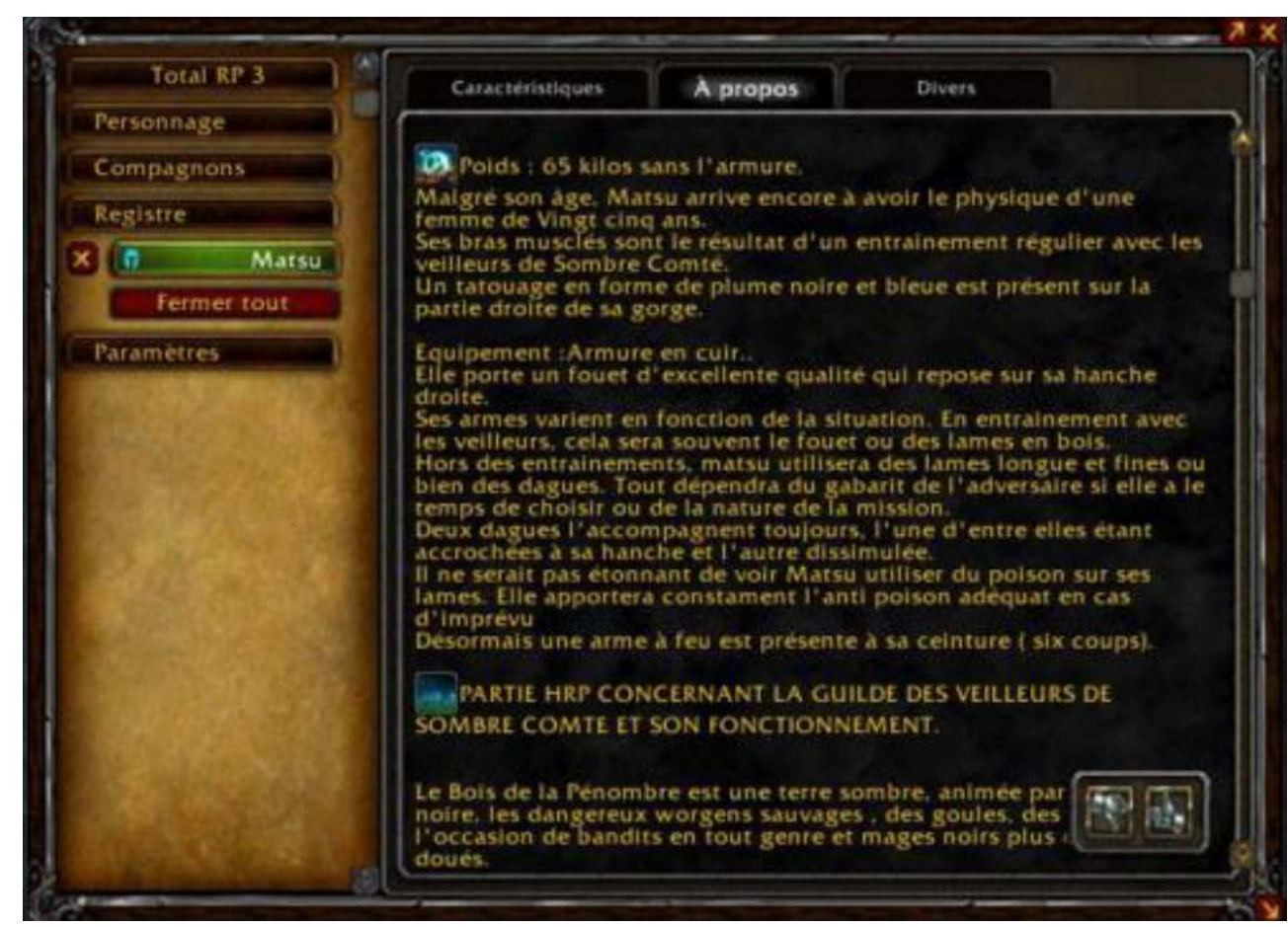

La fiche du personnage d'un joueur (capture d'écran du joueur)

Comme le décrit France Vachey, les différentes fiches de renseignement informent sur :

le nom de famille, l'âge, l'origine, le métier ou activités RP, l'histoire personnelle, la description physique (couleur de peau significative, allure, tatouages, piercings, etc.), le profil psychologique et l'attitude morale, de loyal à chaotique et de bon à méchant, comme l'usage du JdR papier l'a institué (Vachey, 2013, p. 184).

Les fiches de certains joueurs sont importantes à lire avant de parler avec eux, celles-ci justifiant la façon dont ils vont interpréter leur personnage. Le module « Total RP » est ainsi évaluée comme un outil "pratique", voire très utile pour la majorité des répondants au questionnaire dans leur pratique du roleplay.

En dehors et en prolongation du jeu, les forums et les sites internet permettent d'étoffer l'histoire du personnage par l'écriture de textes supplémentaires. Afin d'illustrer et/ou d'enrichir les histoires de leurs personnages, certains rôlistes conçoivent également des supports autres qu'écrits comme des dessins, des figurines ou des machinimas. Une dimension transmédiatique peut être ainsi mise en évidence dans la construction du personnage du roleplay où les joueurs ne semblent pas se limiter au seul support du jeu vidéo.

35 Une fois le personnage créé, il s'agit de l'interpréter. Une règle fondamentale du roleplay est la cohérence de l'histoire du personnage au monde du jeu. Il faut pouvoir correctement « improviser et interagir avec les autres partenaires de ce mode de jeu par 
le biais de dialogues, créatifs, correctement écrits et fidèles à la logique fictionnelle du monde, ainsi qu'aux dynamiques existentielles des protagonistes en présences » (Vachey, 2013, p. 182). D'après le questionnaire, l'histoire des personnages des rôlistes du serveur est principalement liée à celle du jeu mais pas forcément à celle de la communauté où les avis sont partagés, malgré le fait que plusieurs joueurs admettent construire l'histoire de leur personnage au fil des discussions avec ceux des autres. Ce manque de liens peut s'expliquer par le fait qu'ils accordent moins d'importance à la cohérence de l'histoire du personnage avec celle de la communauté qu'avec celle du jeu, qui parait quant à elle tout à fait primordiale.

36 Cette exigence de cohérence amène certains rôlistes à rejeter toute forme de jeu de rôle décalé du Lore. Au cours de l'immersion participante, j'ai moi-même été confrontée à cette exigence; par exemple, un joueur m'a conseillé de changer de cheval car le mien était considéré comme « hors Lore » : il était d'apparence spectrale et ne convenait pas au personnage que j'interprétais. En outre, j'ai intégré une guilde dont une partie des membres se base sur l'histoire d'un univers fictif inventé, soit une partie « hors-Lore ». Ce dernier cas reste toutefois rare et n'est pas à généraliser. Ce genre de « déviances » peut être plus particulièrement observé dans les serveurs privés où les joueurs peuvent remodeler le design du jeu pour qu'il corresponde à leurs histoires. Dans un serveur tel que Kirin Tor, les joueurs basant leur jeu de rôle sur un autre univers fictif sont généralement critiqués.

37 Si l'histoire des personnages des rôlistes doit bien s'accorder avec celle du jeu, ces derniers doivent également adapter leur jeu de rôle dans le cas où l'histoire du Lore change. L'histoire de Warcraft est très ancienne et depuis la sortie du MMORPG en 2004, de nombreuses éditions se sont succédé. Comme le mentionne Laurent Di Filippo (2012), les mondes virtuels sont en constante évolution et l'identité narrative du personnage peut changer "tout en gardant continuité et cohérence». L'extrait de conversation suivant illustre le changement de roleplay d'une guilde que j'ai intégrée lors de mon ethnographie virtuelle :

Tableau 2. Extrait anonyme de conversations entre l'auteur et un joueur

4/19 $22: 31: 44.992 \mathrm{~N}$ chuchote : Vi ! Ici on sait la date de sortie de l'extansion donc on va pouvoir réfléchir à comment on va évoluer

4/19 $22: 31: 52.456 \mathrm{~N}$ chuchote : Vu que notre zone a vu son histoire changée

4/19 $22: 32: 27.872$ À $\mathrm{N}:$ ah bon ? et le décor reste le même?

4/19 $22: 32: 51.346 \mathrm{~N}$ chuchote : Oui, mais en fait, les veilleurs (pnj)..on découvre qu'ils sont à la solde de la légion ardente (les bigs méchants)

4/19 $22: 33: 15.036$ À N : ah mince, ça change beaucoup ça

4/19 $22: 33: 33.262 \mathrm{~N}$ chuchote : Oui, du coup, on va organiser la résistance de sombre-comté

Afin que leurs personnages évoluent dans le monde fictif, le MMORPG doit proposer un univers fictionnel cohérent et vraisemblable. La cohérence et la vraisemblance constituent des paramètres clés dans la fiction, sans quoi le joueur ne pourrait totalement s'immerger dans le monde du jeu. La contrainte de cohérence fonctionne dès lors dans les deux sens, où à la fois l'histoire des rôlistes doit s'accorder à celle du jeu et, en contrepartie, le jeu doit fournir un monde cohérent et vraisemblable dans lequel les rôlistes peuvent y construire leurs personnages. 

primordial à jouer dans l'exercice de celle-ci : «Pour pouvoir communiquer et rester immergé dans son rôle, il est important qu'il n'y ait pas de rupture dans l'univers imaginaire partagé. Cet univers partagé nécessite une culture commune et des conventions communes ", partagées entre les membres de la communauté (Lelièvre, 2012 b , p. 101). À travers les deux points précédents, nous avons pu en effet constater la création de nouvelles règles surplombant celles du jeu pour pratiquer le roleplay dans le serveur. Elles sont apparues au fur et à mesure des interactions entre les joueurs et ont contribué à l'émergence d'une culture commune. Comme tout débutant dans la pratique du roleplay et dans le jeu, j'ai dû moi-même prendre connaissance de ces règles et maitriser les codes établis par la communauté pour interpréter mon personnage, sous peine d'ignorance voire même d'exclusion de la communauté. Plus de $75 \%$ des répondants du questionnaire accordent une grande importance au respect des règles.

Les rôlistes créent régulièrement des événements afin d'animer le monde virtuel. L'organisation d'événements est l'activité principale de la communauté de Kirin Tor: s'y 
déroule des célébrations (mariages, enterrements, hommages), des soirées (œuvres caritatives, fêtes), des jugements (tribunal, Cour) ou encore des combats (duels, compétitions, tournois). Ces événements enrichissent les histoires des personnages et les inscrivent dans une histoire plus vaste, plus globale, celle de la communauté toute entière. Le forum de cette dernière ${ }^{3}$ donne un aperçu de la façon dont ils sont organisés. Quelques rôlistes ou guildes RP se regroupent et conçoivent un scénario roleplay avec une intrigue. Ils la présentent sous forme de lettres, de parchemins, de livres, d'affiches, etc. Ils expliquent en HRP comment l'événement se déroulera et les règles à respecter. Le forum contient également de nombreux contenus narratifs relatant et prolongeant l'histoire globale du jeu ainsi que des discussions autour de l'univers et de la pratique du roleplay.

Par l'entremise d'événements organisés par la communauté, les discussions entre les rôlistes et le forum, des histoires sont constamment inventées. Dû à l'exigence de cohérence entre la première et la troisième narrativité, celles-ci étoffent considérablement l'histoire du jeu. Les guildes RP constituent une dynamique importante dans cette expansion du Lore. Elles se distinguent des guildes HRP du fait qu'elles possèdent une histoire. Elles regroupent des « gens qui ont l'envie de faire un style de rp bien défini, que ce soit être criminel, pirate, garde ou même simplement un groupe d'aventurier » (Extrait de conversation avec un joueur, 15/02/16). Certaines d'entre elles sont intégrées dans la communauté, comme les guildes de religieux, de mercenaires ou de nobles, tandis que d'autres sont « renfermées » sur elles-mêmes. Dans un cas comme dans l'autre, et à quelques exceptions près pour les guildes hors Lore, elles sont liées à l'histoire du jeu. L'extrait de conversation suivant prend l'exemple de la guilde des Veilleurs, pleinement intégrée dans l'histoire du jeu et de la communauté.

Tableau 3. Extrait anonyme de conversations entre l'auteur et des joueurs de la guilde des Veilleurs

2/15 $00: 11: 09.976$ N-KirinTor : Dans l'univers de WoW, dans son histoire depuis Warcraft 2, les Veilleurs sont en fait les gens d'une ville abandonnée par le royaume humain, qui ont décidé de se réunir pour protéger leur ville et faire respecter les lois

2/15 $00: 11: 33.290$ Z-KirinTor : C'est une petite police municipale, en gros.

2/15 $00: 11: 49.574 \mathrm{~N}$-KirinTor : Des pecnots qui ont que ça à faire de leur vie

2/15 $00: 12: 11.300$ Tsukiyocho-KirinTor : et est ce que vous interagissez alors avec les autres personnages du jeu, en dehors de la guilde?

2/15 $00: 12: 30.555$ W-KirinTor : Oui !

2/15 $00: 12: 50.807$ Tsukiyocho-KirinTor : en tenant votre rôle de veilleur? (ma question supposait ceci)

2/15 $00: 12: 52.614$ N-KirinTor: Bien sûr! On est une guilde de "garde", on se base, normalement, sur l'interaction rp avec les autres joueurs

2/15 $00: 13: 17.526$ Z-KirinTor: Oui. On peut faire du rp 'sauvage' (Avec des personnes hors guildes) ou même de l'inter-guilde (en jouant avec une autre guilde )

2/15 $00: 14: 01.422$ Tsukiyocho-KirinTor : oui ok :)

Chez les Veilleurs, comme c'est le cas pour d'autres guildes RP, les membres se réapproprient le Lore. Tel que les joueurs le mentionnent ci-dessus, à l'origine, les Veilleurs sont des PNJs, qui ont une histoire inscrite dans la genèse de Warcraft. Les 
rôlistes se sont inspirés de leur histoire et en ont fait une guilde. En plus de s'être réapproprié le Lore, ils se sont emparés de la ville de Sombre-Comté, le quartier général des Veilleurs, de ses bâtiments et de ses environs, pour exercer leur jeu de rôle (cf. Figure 3).

Figure 3

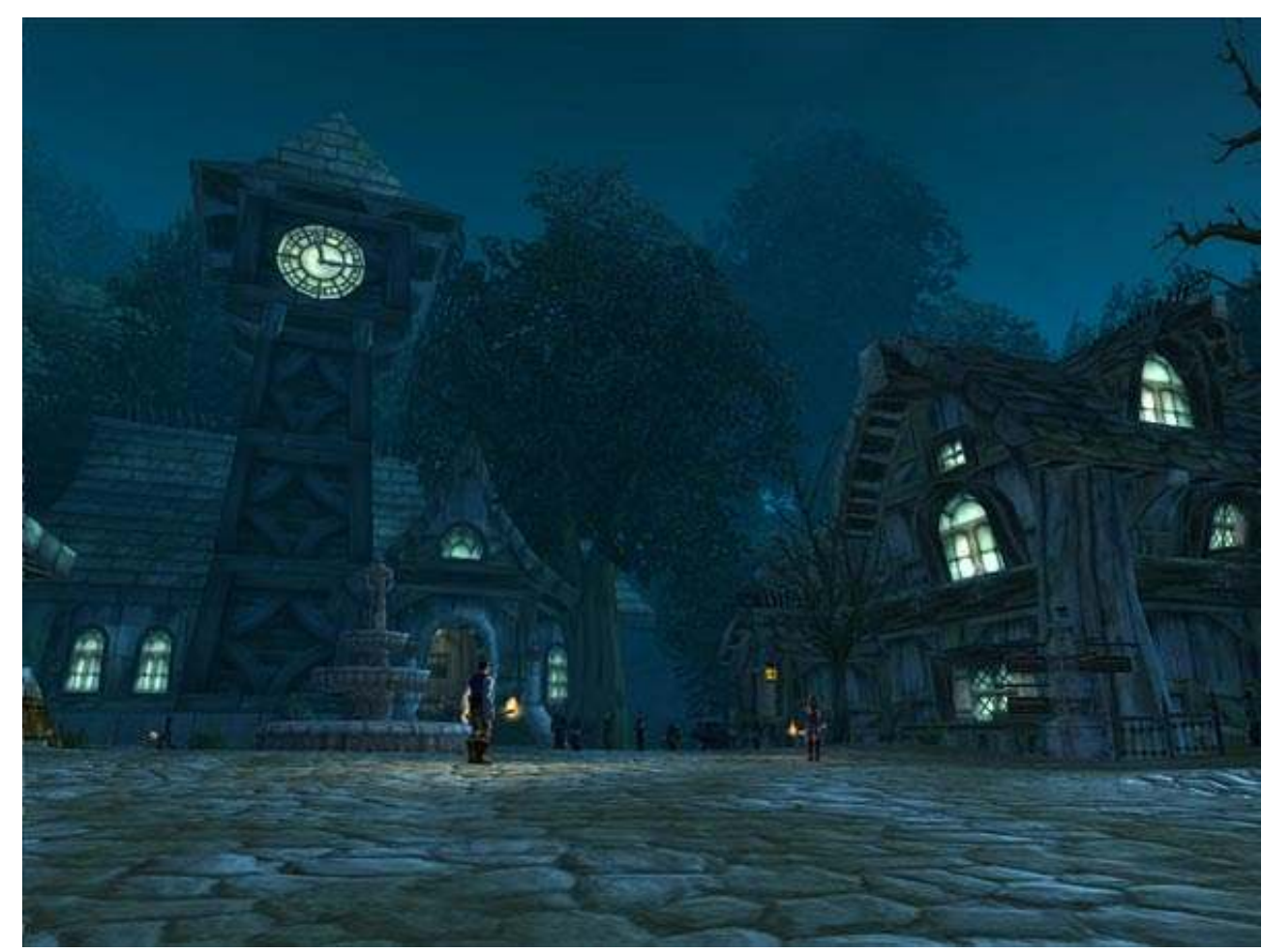

La ville de Sombre-Comté (WikiWoW)

La communauté se réapproprie également certains éléments du jeu lors des événements qu'elle organise : les rôlistes investissent les lieux et les bâtiments du jeu avec leurs propres histoires, voire même détournent leur histoire pour qu'elle corresponde à leur jeu de rôle. Les PNJs ont aussi un rôle à jouer dans la pratique du roleplay: le système du jeu permet de parler à leur place, ce qui explique que certains joueurs les utilisent à l'occasion d'événements pour prendre la parole et avoir l'attention du public. De plus, les PNJs sont souvent intégrés dans les histoires des rôlistes, aussi bien à l'occasion de discussions, d'événements que dans leur historique.

La première comme la seconde narrativité peut être par conséquent mobilisée par la communauté des rôlistes. D'une part, ceux-ci vont puiser dans l'histoire du jeu pour construire leurs personnages et former l'identité de la communauté. D'autre part, ils vont interagir avec les différents espaces du jeu et les PNJs pour développer leur jeu de rôle. Le jeu vidéo propose toutefois peu d'infrastructures pour la pratique en elle-même, si bien que les rôlistes ont tendance à créer leurs propres outils pour pouvoir interagir avec la communauté. Un premier exemple a été évoqué en mentionnant le forum de la communauté, particulièrement utile pour l'organisation d'événements. Le module complémentaire «Total RP » en est une seconde illustration : il aide non seulement à fabriquer son personnage par l'élaboration de diverses fiches, mais facilite aussi l'interaction avec les autres rôlistes par les informations qu'il leur fournit. Le module 
propose en outre d'activer un mode RP (phase rôlistique) ou HRP (hors-roleplay), en fonction de l'interaction en tant que personnage ou joueur.

En résumé, plus qu'interagir avec le jeu, les rôlistes participent à son histoire en l'enrichissant constamment à travers leurs activités en jeu et en dehors du jeu (création de nouvelles règles, organisation d'événements, rédaction d'histoires, conversations entre personnages, etc.). Cette participation provoque un changement dans la position tenue par les rôlistes dans le jeu. Dans les œuvres numériques, «Le récepteur est ici à la fois joueur (acteur) et spectateur : il adopte simultanément une posture d'action ludique et une posture d'attention esthétique; il contemple et joue en même temps » (Schaeffer, 1999, p. 334). Dans le cas de la pratique du roleplay dans le MMORPG, les joueurs sont à la fois spectateur/lecteur, auteur, critique et concepteur. Ils forment par conséquent une communauté participative, leurs récits formant une fiction participative où "s'y déroule une action totalement virtuelle, au sens où elle ne procède que dans l'espace imaginaire collectif» (Vachey, 2013, p. 180).

\section{Conclusion}

49 L'approche narratologique s'est rapidement révélée insuffisante concernant les formes interactives du récit du jeu vidéo, ses approches étant » majoritairement menées à partir du critère de l'action et du scénario, celles qui privilégiaient le level design et qui observaient le jeu vidéo de l'extérieur » (Marti, 2012, p. 12). Trouvant son origine dans les travaux de Johan Huizinga (1938) et de Roger Caillois (1967) sur le jeu, « la ludologie s'est affirmée comme une science des jeux prenant en compte l'organisation de la pratique ludique (espace, temps, règles), mais aussi sa dimension sociale (position du joueur, effets du jeu) » (Marti et Baroni, 2014, \$14). Le croisement de ces deux disciplines a permis de prendre en compte le rôle du joueur dans l'actualisation du récit du jeu vidéo.

50 Au regard de l'évolution des théories narratives sur le jeu vidéo, peu se sont malgré tout intéressées à l'interaction de la narrativité vidéoludique avec les créations narratives de la communauté des joueurs. La recherche présentée dans cet article répond à ce manque en confrontant les trois narrativités relevées dans le MMORPG par l'étude de la pratique du roleplay. Les résultats mettent en avant une interaction importante, si ce n'est même contraignante, entre la première et la troisième narrativité, soit entre l'histoire globale du jeu et les histoires créées par les rôlistes. D'un côté, le monde du jeu doit proposer un univers cohérent et vraisemblable dans lequel les joueurs peuvent y développer leurs personnages. D'un autre côté, les rôlistes doivent construire et interpréter leurs personnages en tenant compte de l'histoire globale du jeu. Quant à la seconde narrativité, celle issue de l'interaction du joueur avec le jeu, les rôlistes de la communauté observée tentent de s'en émanciper le plus possible, particulièrement dans le cadre du mode divin, par la création de leurs propres règles et infrastructures.

51 Cette émancipation vis-à-vis de la seconde narrativité amène les rôlistes à affirmer d'autant plus leur lien avec la substance narrative du jeu-même. Plutôt que développer un jeu au-dessus du jeu, ils inscrivent leur jeu de rôle dans l'histoire du jeu. Bien que certaines activités de roleplay soient plutôt de l'ordre du « méta », comme c'est le cas pour la création de règles supplémentaires, la gestion de sites internet et l'organisation des guildes, ces activités reposent sur le jeu lui-même et garantissent le lien avec la première narrativité. Construites autour d'une pratique telle que le roleplay, ces activités contribuent à l'investissement des joueurs dans la diégèse. 
52 En outre, les résultats soulignent l'importance du rôle de la communauté RP dans la configuration narrative du MMORPG. La communauté a la particularité de s'emparer des brèches du récit de WoW pour les combler avec les histoires de ses membres. Elle s'approprie également l'espace du jeu, en utilisant les bâtiments et en leur donnant des significations supplémentaires, voire totalement nouvelles. Elle mêle ainsi la représentation du jeu à la fiction. Ces constats amènent à s'interroger sur la position du joueur dans le jeu (qui devient l'auteur d'un récit) et contribuent à la réflexion sur le phénomène de la réappropriation.

Selon Henry Jenkins (2013), les nouveaux médias actuels renforcent cette idée de participation à la culture (s'opposant à celle de la passivité du spectateur), si bien qu'« on ne parle plus de publics mais de communautés participatives, élaborant des œuvres, des appendices critiques, des tactiques informationnelles: pages perso, forums, fanfics, vidéos, tweets, mèmes » $(p .7)$. Une culture participative peut être ainsi relevée à travers la pratique du roleplay dans les MMORPGs où les joueurs créent des récits à travers différentes activités, contribuant à l'histoire même du jeu et l'enrichissant notablement. Le roleplay en ligne s'inscrirait ainsi dans ce passage de l'interactivité à la participation, cette pratique n'étant pas initialement prévue par les producteurs du jeu.

Dans une recherche future, il serait intéressant d'investiguer dans les serveurs privés de WoW, ceux-ci pouvant être personnalisés par les joueurs et mettant probablement en avant un détournement plus important du jeu. Les serveurs américains présentent aussi des différences sur la manière dont le roleplay est pratiqué, si bien que la pratique pourrait être reliée à la culture des pays. En outre, cet article s'est consacré spécifiquement à la forme écrite du roleplay. Or, dans le serveur de Kirin Tor, des joueurs réalisent du roleplay « vocal » à l'aide de logiciels de communication tels que Teamspeak ou Mumble, une pratique qui apparait d'ailleurs de plus en plus dans d'autres mondes virtuels et sur laquelle il serait intéressant de se pencher. Une dernière piste serait de s'interroger sur les notions d'" habiter" et d'" habitat ", et se demander dans quelle mesure les personnages des rôlistes pourraient « habiter » le monde du jeu (Lucas, 2013).

\section{BIBLIOGRAPHIE}

AMATO E. A. (2007), « Quelle ethnométhodologie appliquée aux jeux vidéo multijoueurs persistants?», Cahiers d'ethnométhodologie, $\mathrm{n}^{\circ} 1$, pp. 23-45.

BARNABÉ F. (2012), Narration et jeu vidéo : pour une exploration des univers fictionnels, mémoire de recherche, Université de Liège.

BERRY V. \& BROUGERE G. (2001), « Play and virtual communities », 2nd ICCP World Play Conference, Erfurt (RFA), 6-8 juin, https://experice.univ-paris13.fr/membres/enseignantschercheurs/gilles-brougere/publications-et-communications-de-gilles-brougere/.

BERRY V. (2012a), L'expérience virtuelle. Jouer, vivre, apprendre dans un jeu vidéo, Rennes, Presses universitaires de Rennes. 
BERRY V. (2012b), « Ethnographie sur Internet : rendre compte du "virtuel“ », Les Sciences de l'éducation - Pour l'Ère nouvelle, vol. 45, n 4, pp. 35-58, https://www.cairn.info/revue-les-sciencesde-l-education-pour-l-ere-nouvelle-2012-4-page-35.htm

BOELSTORFF T., NARDI B.A., PEARCE C. \& TAYLOR T.L. (2012), Ethnography and Virtual Worlds. A Handbook of Method, New Jersey, Princeton University Press.

BOELLSTORFF T. (2013), Un anthropologue dans Second Life. Une expérience de l'humanité virtuelle, Louvain-la-Neuve, Academia-L'Harmattan.

BREMOND C. (1973), Logique du récit, Paris, Seuil, coll. « Poétique ».

CAILLOIS R. (1967 [1958]), Les jeux et les hommes : le masque et le vertige, Paris, Gallimard.

COLERIDGE S. T. (1817), Biographia literaria, http://www.english.upenn.edu/ mgamer/Etexts/ biographia.html

DAJEZ F. (2006), « La figurine interfacée, à propos de l'Odyssée d'Abe » in P. Barboza \& J.-L. Weissberg (dir.), L'image actée : scénarisation numérique, parcours du Séminaire. L'Action sur image, Paris, L'Harmattan, pp. 23-24.

DANNENBERG H. (2004), « Ontological Plotting: Narrative as a Multiplicity of Temporal Dimensions ", in J. Pier (ed.), The Dynamics of Narrative Form. Studies in Anglo-American Narratology, Berlin, Walter de Gruyter, pp. 159-189.

DI FILIPPO L. (2012), « Les notions de personnage-joueur et Roleplay pour l'étude de l'identité dans les MMORPG », Interrogations, n 15, http://www.revue-interrogations.org/Les-notions-depersonnage-joueur.

ECO U. (1984), The Role of the Reader, Bloomington, Indiana University Press.

Encyclopédie des Royaumes Renaissants (2010), Hors-roleplay, http://www.le317.fr/guide/ index.php?title=Hors-roleplay.

FORTIN M.-F. (1996), Le processus de la recherche : de la conception à la réalisation, Montréal, Décarie.

GENVO S. (2009), Le jeu à son ère numérique : comprendre et analyser les jeux vidéo, Paris, L'Harmattan.

HUIZINGA J. (1951 [1938]), Homo Ludens essai sur la fonction sociale du jeu, Paris, Gallimard..

JENKINS H. (2002), « Game design as narrative architecture », in N. Wardrip-Fruin \& P. Harrigan (eds.), First Person: New Media as Story, Performance, and Game, Cambridge, MA, The MIT Press, pp. 118-130.

JENKINS H. (2013), La culture de la convergence. Des médias au transmédia, Paris, Armand Colin.

KRICHANE S. (2014), « L'intrigue en trois dimensions », Cahiers de Narratologie, 27, https:// narratologie.revues.org/7014.

LELIÈVRE E. (2012a), « Roleplaying as a creation form and a source of inspiration in RPG virtual », in S. Bornhofen, J.-C. Heudin, A. Lioret \& J.-C. Torrel (eds.), Virtual worlds, Artificial Ecosystems \& Digital Art Exploration, Science-eBook, http://www.armaghia.fr/wp-content/uploads/2012/04/ roleplaying.pdf.

LELIÈVRE E. (2012b), Des jeux de rôle en ligne tridimensionnels aux jeux à réalité alternée : expérience esthétique, création et expérimentation, thèse de doctorat, Université Paris 8.

LUCAS J.-F. (2013), De l'immersion à l'habiter dans les mondes virtuels : le cas des villes dans Second Life, thèse de doctorat, Université Rennes 2. 
MARTI M. (2012), « Jeux vidéo et logiques narratives », in H. T. Minassian, S. Rufat \& S. Coavoux, Espaces et temps des jeux vidéos, Questions théoriques, pp. 73-91.

MARTI M. (2014), « La narrativité vidéoludique : une question narratologique », Cahiers de Narratologie, 27, https://narratologie.revues.org/7009.

MARTI M.\&t BARONI R. (2014), « De l'interactivité du récit au récit interactif », Cahiers de Narratologie, 27, https://narratologie.revues.org/7077.

MILLENIUM (2015), Index of World of Warcraft, http://www.millenium.org/wow/accueil/Guide/ index-world-of-warcraft-lexique-des-termes-les-plus-utiles-du-jeu-leur-definition-et-nosarticles-correspondants-124035

NARDI B. A. (2010), My Life as a Night Elf Priest. An Anthropological Account of World of Warcraft, Ann Arbor, The University of Michigan Press.

PEARCE C. (2011), Communities of Play: Emergent Cultures in Multiplayer Games and Virtual Worlds, Cambridge, The MIT Press.

RYAN M.-L. (2001), Narrative as Virtual Reality: Immersion and Interactivity in Literature and Electronic Media, Baltimore, Johns Hopkins University Press.

SCHAEFFER J.-M. (1999), Pourquoi la fiction ?, Paris, Éd. du Seuil.

SERVAIS O. (2012), « Autour des funérailles dans World of Warcraft. Ethnographie entre religion et mondes virtuels », in J.P. Delville (dir.), Mutations des religions et identités religieuses, Paris, Mame-Desclée, pp. 231-252.

SERVAIS O. (2013), Un anthropologue dans Second Life. Une expérience de l'humanité virtuelle, Louvainla-Neuve, Academia-L'Harmattan.

TAYLOR T. L. (2009), Play Between Worlds: Exploring Online Game Culture, Cambridge, The MIT Press.

TREMEL L. (2003), «La pratique du jeu vidéo : un objet d'études sociologiques ? » in M. Roustan (dir.), La pratique du jeu vidéo : réalité ou virtualité ? Paris, L'Harmattan, pp. 157-169.

VACHEY F. (2013), « Rapports identitaires et narratifs en ligne », in E. A. Amato \& E. Perény (dir.), Les avatars jouables des mondes numériques : théories, terrains et témoignages de pratiques interactives, Paris, Lavoisier, pp. 177-204.

WUYCKENS G. (2016), L'univers narratifétendu des MMORPGs. Du Lore au roleplay : pour une étude de la narrativité du MMORPG, mémoire de recherche, Université Catholique de Louvain.

\section{NOTES}

1. Le mot étant communément utilisé en anglais par la communauté des joueurs de jeux vidéo, le terme "roleplay » est préféré à celui de "jeu de rôle ». D'autres raisons justifient son emploi en anglais : d'une part, le jeu de rôle est souvent associé au jeu de rôle papier, sur forum ou le grandeur nature (GN), et non au jeu de rôle tel que pratiqué dans les MMORPGs. D'autre part, le MMORPG est un "roleplaying game ", c'est-à-dire, traduit littéralement en français, un jeu de jeu de rôle. Ce jeu propose en effet dès le début aux joueurs de jouer un rôle à travers leur personnage et son évolution dans le jeu. Le personnage incarné à travers le jeu est toutefois bien différent de celui créé par les rôlistes, d'où la préférence pour le terme « roleplay».

2. «Sont qualifiés de hors-roleplay tous les propos et discussions portant sur le jeu mais qui ne sont aucunement prononcés par les personnages" (Encyclopédie des Royaumes Renaissants, 2010). 
3. Forum de la communauté RP du serveur Kirin Tor : http://kirintor-rp.forumsrpg.net/f7evenements

\section{RÉSUMÉS}

L'article s'intéresse à l'architecture narrative des MMORPGs, les jeux vidéo de rôle en ligne massivement multijoueur. Il les caractérise par une triple narrativité : la narrativité préétablie par les concepteurs du jeu, la narrativité issue de l'interaction du joueur avec le jeu et la narrativité créée par la communauté des joueurs. Par l'intermédiaire d'une ethnographie virtuelle menée dans le jeu World of Warcraft, l'article vise à comprendre la configuration spécifique que peuvent prendre ces trois narrativités dans les pratiques communautaires observées du roleplay ou jeu de rôle. Les résultats mettent en avant une interaction importante entre la première et la troisième narrativité, soit entre l'histoire du jeu et l'histoire des personnages interprétés par les rôlistes. Quant à la seconde narrativité, les rôlistes tentent de s'en émanciper le plus possible, par la création de leurs propres règles et infrastructures pour pratiquer le roleplay.

The paper examines the narrative architecture of MMORPGs, or massively multiplayer online role-playing games. It characterizes them by a triple narrativity : the narrativity pre-established by the game designers, the narrativity resulting from the player's gameplay and the narrativity created by the community of the players. Through a virtual ethnography conducted in the game World of Warcraft, the paper aims to understand the specific configuration that these three narrativities can take in the observed community roleplay practices. The results highlight an important interaction between the first and the third narrativity, that is, between the storyworld and the story of the characters interpreted by the role-players. As for the second narrativity, the role-players try to emancipate themselves from it as much as possible, by creating their own rules and infrastructures to practice role-play.

\section{INDEX}

Keywords : narrativity, roleplay, MMORPG, narrative, story, virtual community.

Mots-clés : narrativité, jeux de rôle, jeux de rôle en ligne massivement multijoueur (MMORPG), récit, histoire, communauté virtuelle

\section{AUTEUR}

\section{GÉRALDINE WUYCKENS}

Université Catholique de Louvain 